\title{
Pterodrone: a pterodactyl-inspired unmanned air vehicle that flies, walks, climbs, and sails
}

\author{
S. Chatterjee ${ }^{1}$, B. Roberts ${ }^{2} \&$ R. Lind ${ }^{2}$ \\ ${ }^{1}$ Museum of Texas Tech University, USA \\ ${ }^{2}$ Mechanical and Aerospace Engineering, University of Florida, USA
}

\begin{abstract}
Bio-inspiration has led to a variety of robotic designs, especially small Unmanned Aerial Vehicles (UAVs) for urban environment that have taken cues from birds, bats, and insects. However, one group of extinct flying animals, the pterosaurs that flew over the heads of dinosaurs, have been largely overlooked in this search for biological solutions to mechanical problems. This project will develop and demonstrate a next-generation capability for sensor emplacement using pterosaurs as the model animal. Tapejara wellnhoferi, a pterodactyloid from the Early Cretaceous of Brazil, shows promise to develop an UAV (Pterodrone) of much superior range, a sensor platform capable of aerial, terrestrial, and aquatic locomotion by altering its wing shape. Tapejara was a sophisticated flyer with a cruising speed of $30 \mathrm{~km} / \mathrm{hr}$. The cranial sail led to the design of a vertical tail at the nose of the Pterodrone that varies both longitudinally and vertically, as opposed to the more conventional, fixed aftlocated vertical tail. The novel forward-placed tail design shows a marked improvement in the allowable turning radius when flight vehicle capabilities are normalized to constant thrust or power. Fabricated of lightweight carbon fiber, the Pterodrone employs a flexible wing design to achieve a wide range of multimodal locomotion. On land, the Pterodrone could walk quadrupedally for gathering information. Wheel-pod, a new device, employs the retractable foot on demand for terrestrial locomotion, and is flexibly hinged to the rear wheel, with a gecko-like foot that permitted vertical landing on the wall. In aquatic environments, the Pterodrone achieves the configuration of a two-masted catamaran sailboat, where the cranial sail acts as a jib and the two upright wings function as a pair of mainsails to catch the bridge. The sternum and hindlimbs would have contacted the water much as trimaran's hulls do to provide stability.
\end{abstract}


The enhanced agility of the Pterodrone, a combination of sea-air-ground robotics, with embedded sensing elements will allow operations throughout urban areas, even buildings or dangerous and inaccessible environments for search and surveillance. With a wide range of multimodal locomotion, the Pterodrone is a vehicle with mission capability that would gather data from sights, sounds, and smells in urban combat zones and transmit information back to the ground station in real-time.

Keywords: Pterodrone, pterodactyl-inspired robots, Tapejara, forward placement of vertical tail, aerial turning, multimodal locomotion.

\section{Introduction}

Considerable interest has been generated in recent years in designing UAVs to navigate through a dense field of obstacles and maintaining efficiency while sensing. The UAVs are remotely piloted or self-piloted aircraft that can carry cameras, sensors, communication equipment or other payloads. They have been used in reconnaissance and intelligence-gathering roles since the 1950s, and more challenging roles are envisioned, including combat missions. The high level of current interest in UAVs is the result of the nearly simultaneous emergence of their technological feasibility and an array of compelling new military needs, especially in urban environments. These vehicles have both potential to remove the risk of loss of human life from aerospace applications and eliminate the many system requirements necessary to accommodate humans. Thus UAVs can be made more agile to make tighter turns than a human could withstand, made smaller than a size that could carry humans, and filled with many more military payload or sensor systems than the same sized manned vehicle could carry. These opportunities for advanced mission capability reach a pinnacle in the use of a new class of flight vehicles, the Micro Air Vehicles (MAVs) by the Defense Advanced Research projects Agency (DARPA), which are less than $15 \mathrm{~cm}$ in length, width, or height.

Recent developments in the study of UAVs for urban environment navigation and sensing have used biological systems for inspiration. Morphing vehicles have taken cues from birds [1], bats [2], and insects [3] for their configuration, materials, and flight maneuvers. Flapping vehicles have become more commonplace as researchers look to birds, bats, and insects to create enough lift to carry the necessary payloads and to sufficiently control the vehicle for flight. However, one group of extinct flying animals, the pterosaurs, has been largely overlooked in this research for biological solutions to mechanical problems. The pterosaurs show promise as a model for not only a capable flight vehicle, but as a small functional air-ground sea robotic, named Pterodrone, with a wingspan of $1.4 \mathrm{~m}$ and weighs only $0.4 \mathrm{~kg}$ that will provide autonomous surveillance targeting, tagging and biochemical sensing across a variety of operation areas. Pterodrone displays a wide variety of configurations, depending on specific mission requirements. It is about the size of the smallest current missionized UAV, the Sender, developed and operated by Naval Research Laboratory. 


\section{Biologically-inspired aircraft design}

\subsection{The approach}

Biomimetics is a new way of viewing and valuing nature, studying its best ideas and then imitates these designs and processes to solve human problems. Nature has innovated countless extraordinary designs through million years of evolution and has learned what works and what lasts and has rejected flawed attempts. The natural structure provides a clue to what is useful in a mechanism. Flight of animals must be one of the most novel and complex designs in nature that has inspired the invention of aircraft. Biologically-inspired approaches have certainly been used for a wide variety of robotic designs, which could be deployed in places where people would be too conspicuous or at risk of physical harm. The extensive study into morphing by DARPA and NASA noted that birds increase efficiency by varying shape throughout the flight envelope. The entire field of MAVs, including the newest investigations into flapping-wing systems, is strongly influenced by designs that mimic the wing characteristics and associated flow properties of birds and insects. In each case, the concepts focus on efficiency for a single mode of locomotion even though many natural systems have reasonable capabilities for multiple modes of locomotion.

The Pterodrone project will implement concepts from biology that are specifically well suited for sensor emplacement. In particular, the configurations which nature has shown as effective for locomotion across air, land, and sea will be critical to achieving the mission capability of interest to the warfighter. The actual efficiency of such locomotion in these diverse modalities will obviously be considered although a reduced efficiency in any modality will be acceptable if balanced by an unprecedented ability for locomotion across all four modalities: flying, running, climbing, and sailing. The evolution of biological systems to achieve stunning levels of mobility can advance robotics far beyond the current levels which are optimized for, and limited to, a single regime of air, land, or sea.

\subsection{Pterosaurs: biological inspiration}

Pterosaurs, the extinct volant archosaurs of the Mesozoic that flew over the heads of dinosaurs, have fascinated scientists, the general public, and the engineering community for almost 200 years. Pterosaur flight is an intriguing topic that stimulates imagination, creativity, and appeals to the scientific and engineering community as well the general public. Collaborations between paleontologists and aeronautical engineers have a long history since 1914 to unravel the mystery of the flight dynamics of pterosaurs. Pterosaurs were the first radiation of vertebrates that developed active, powered flight. They have a unique wing anatomy unlike that of birds and bats, wherein the forelimb and the hyper-elongated fourth finger formed a single bony spar at the leading edge to support a long and narrow membranous wing like that of a sail. Contrary to the thin, compliant, and uniform bat wing, the skin membrane in pterosaurs was not mechanically homogenous structure, but had a relatively elastic section in 
the inner wing (plagiopatagium) and a stiffer semirigid outer wing (dactylopatagium); the latter is reinforced by densely packed, radiating rod-like fibers - actinofibrils - that conferred structural integrity and allowed folding and unfolding of the wing like the battens of a sail to reduce the stretch. This unique variable wing structure gives pterosaurs more options for flight than most birds and bats. Pterosaurs had a dense covering of hair distributed over most of the head, body, base of the tail, and upper part of the arms and legs that provided insulation and streamlining of the body surface.

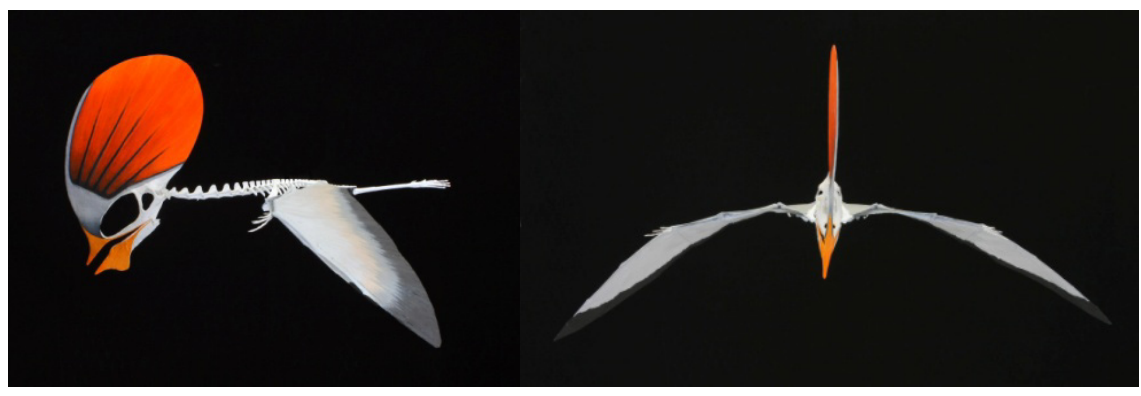

Figure 1: Skeletal reconstruction of Tapejara wellnhoferi, an Early Cretaceous pterodactyloid from Brazil in flying posture, the biological inspiration for the Pterodrone: left, left lateral view; right, front view.

Unlike birds and bats, the pterosaurs, which were equally sophisticated flyers, have received little attention for creating biomimetic models. This is mainly because, until recently, the available fossil material has not been of sufficient quality to permit an accurate three-dimensional reconstruction of the skeletal anatomy: a prerequisite for a biomimetic model. One of the remarkable pterosaurs is a crow-size pterodactyloid from the Early Cretaceous Santana Formation of Brazil, called Tapejara wellnhoferi with a wingspan of $1.28 \mathrm{~m}$ that possessed an aerodynamic oddity - an enormous vertical cranial crest. The skull of Tapejara is relatively short and toothless with a large sagittal premaxillary crest extending to the posterior end of the skull, an expanded nasoantorbital fenestra that occupies one-third of the lateral surface of the skull, a ventrally inclined tapering rostrum, and a mandibular sagittal crest. The premaxillary crest was lightly built and supported at its posterior edge a flaring membranous structure like a vertical sail, which was reinforced internally by stiff fiber-like networks of cornified epidermis. The fibers in the soft membranous tissue provided the structural framework of the cranial sail that could be traced forward into the bony part of the crest to maximize the strength and stiffness. The semirigid cranial sail would be about 16 times the lateral surface of the premaxillary crest and must have had an aerodynamic effect. Such elaborate and idiosyncratic design of the cranial crest is probably related to courtship and mating display, but its location ahead of animal's center of gravity appears to be an anomaly in flight design (Fig. 1). Several novel stabilizing and control 
surfaces might have compensated for yaw instability and made Tapejara a versatile flyer as it traded off instability for maneuverability. Most likely the cranial sail functioned as a sophisticated wind sensor and acted as an active stabilizer like an automatic feedback control system. It could relay the aerodynamic perturbations to the wing via vestibular apparatus of the inner ear and enhance the aerial turn [4]. Tapejara probably fed on small fish and crustaceans while dipping its short and pointed beak during surface sailing like a two-masted sailing boat with a jib [5].

We have taken one of the biggest challenges to develop a pterodactyl-inspired robotic vehicle, named Pterodrone that is swift and maneuverable, capable of aerial, terrestrial, scansorial, and aquatic locomotion for use in surveillance or search-and-rescue operations [6]. However, pterosaurs make for an ideal model to design a sensory/payload delivery system, rather than bats or birds for several reasons.

1. A primary interest is the scalability of vehicle size. The range of missions for which a UAV may be designed can range from high-altitude environments to urban environments to indoor environments. The variations in size and speed required for the platforms across these environments are not accompanied by an easy scaling of aerodynamics. The mechanisms associated with propulsion for the flapping of birds, bats, and insects is well known to be limited to a small range of Reynolds' number as is the associated range of missions.

The range of specimen size for pterosaurs is relatively large and encompasses the range of platforms often referred to as micro air vehicles, miniature air vehicles, and small air vehicles. As such, designs based on these concepts can utilize the scalability already demonstrated for the aerodynamics to expand the mission capability of the warfighter. Pterosaurs were capable of success in a great variety of size ranges and modes of flight. Pterosaurs thrived for 160 million years along with dinosaurs until the sudden biologic catastrophe that drove much of globe's species to extinction at the end of the Cretaceous. During their time, pterosaurs evolved into a great range of diverse organisms. The body mass of pterosaur ranges from $0.015 \mathrm{~kg}$ to $70 \mathrm{~kg}$ and the wingspan from $0.4 \mathrm{~m}$ to $10.4 \mathrm{~m}$, thus showing a wide range of adaptations in coastal areas [7]. Some Late Cretaceous pterodactyloids such as Quetzalcoatlas became the largest flying animal with wingspan as wide as a Lear jet. Not only did pterosaurs vary greatly in size but also in their methods of locomotion. The smallest pterosaurs were capable of hovering flight, much like hummingbirds of today. The medium-sized pterosaurs adapted for continuous level flight, and the largest pterosaurs were efficient soarers.

2. Pterosaur anatomy is unique, unlike that of bats and birds, and remarkably similar to the structure commonly used in modern small UAVs. Pterosaurs use a leading edge spar to support a membrane that serves as the lifting surface. Unlike the flexible bat wing, the wing membrane of pterosaurs was not a mechanically homogenous structure, but had a relatively elastic inner section and a stiffer distal section, supported by densely packed fine, parallel fibers radiating throughout the dactylopatagium. These fibers are oriented perpendicular to the direction of spanwise tension to maximize the strength and stiffness required 
during flight. The fibers confer some stiffness and structural integrity, probably to control surface curvature for aerodynamic efficiency and to prevent billowing and tearing during flight. These fibers functioned like the ribbings of an umbrella for folding and unfolding the wings when walking or flying. These fibers act much like the carbon fiber used to support the lifting membrane of UAVs, transporting the stresses to the leading edge spar, preventing extreme airfoil shape change, and reducing the likelihood of catastrophic damage to the wing membrane. A further comparison between biological and mechanical flight systems is shown below to demonstrate the aptitude for modeling a small UAV design on pterosaurs.

Table 1: Biological-mechanical flight system comparison.

\begin{tabular}{|l|cccccc|}
\hline platform & $\begin{array}{c}\text { wing } \\
\text { surface }\end{array}$ & $\begin{array}{c}\text { muscle } \\
\text { actuation }\end{array}$ & $\begin{array}{c}\text { flapping } \\
\text { motion }\end{array}$ & $\begin{array}{c}\text { vertical } \\
\text { tail }\end{array}$ & $\begin{array}{c}\text { wing } \\
\text { extenders }\end{array}$ & $\begin{array}{c}\text { structural } \\
\text { elements }\end{array}$ \\
\hline insect & rigid & low & high & no & no & chord-span \\
bird & feather & high & high & no & feathers & span \\
bat & membrane & high & high & no & joints & chord-span \\
pterosaur & membrane & low & low & yes & joints & span \\
MAV & membrane & low & low & yes & joints & span \\
\hline \hline
\end{tabular}

3. The mode of locomotion is closely related to the issue of mission effectiveness. The variety of environments in which the aircraft need to operate introduces considerable challenges. These missions will obviously require flight; however, aspects of sensor emplacement will drive the need for additional modalities. Pterosaurs make even more sense as a model for a multi-locomotive sensory system because they also sailed on the oceans and walked on the ground using a quadrupedal gait, which is much simpler to manufacture and control than the bipedal gait that birds commonly employ. Such multimodal locomotion enables an incalculable range of missions. So, pterosaurs have a proven record of aerodynamic and hydrodynamic success and due to their anatomy and methods of locomotion, serve as a sensible model around which to have a multimodal locomotory and sensory system capable of navigating dense obstacle fields and efficiently sensing their environment. An aircraft based on pterosaur concepts may be able to fly to a rooftop then walk under an overhang to mount a sensor in a dark corner.

\section{Vehicle design and fabrication}

\subsection{Baseline shape of the Pterodrone for flight}

A baseline shape is determined for the Pterodrone. This baseline shape and computational model of the Pterodrone, as shown in Figure 2, represent a stable vehicle that has demonstrated flight capability as a MAV [8]. 


\subsection{Forward-placed vertical tail}

Some pterodactyloids such as Tapejara have a particularly interesting aerodynamic feature that has not been characterized before (Fig. 1). They possessed a large membranous cranial sail along the sagittal plane of the head. While the relative size of the cranial crest and its multitasking function is still being hotly debated, its aerodynamic impact is unavoidable. In some ways the cranial sail must have acted like a vertical tail (Fig. 3). The difference is its placement in front of the center of gravity. Common sense would dictate that such a placement would create large instabilities, as a wind gust from the right would yaw the pterosaur left, thus creating an even larger wind force acting to yaw the pterodactyl further. This begs the question, how did the pterosaurs compensate for the creation of this lateral instability and was there any aerodynamic advantage to placing this aerodynamic surface in front of the center of gravity to justify the instability created?

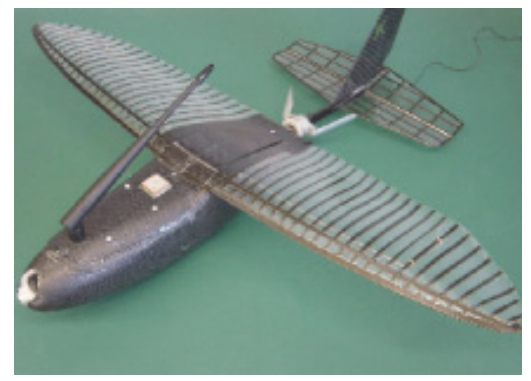

Figure 2: Baseline vehicle in flight configuration.

This project presents a revolutionary change in vehicle design by demonstrating configurations that have never been flown. Specifically, location of the vertical tail of the Pterodrone, mimicking the cranial sail of Tapejara, will range far beyond the standard placements. Consider that the vast majority of the aircraft either have the vertical tail pointing out the top of the rear fuselage or else have no tail whatsoever; conversely, Pterodrone will include a vertical tail that can slide forward/back along the fuselage and up/down through the nose as shown in Figure 3.
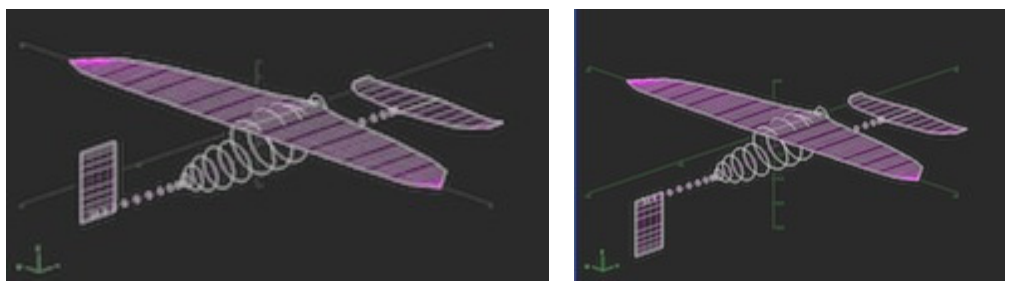

Figure 3: Configuration of Pterodrone with vertical tail at the nose. 
The purpose of a vertical tail is a critical element of any aircraft design. The traditional placement of a vertical tail that points up is chosen to provide static stability in both roll and yaw axes. A tailless vehicle lacks that stabilizing contribution so the wing sweep is increased to provide a stabilizing influence in roll and yaw. In each case, the design is focused on stability.

Pterodrone, with this novel forward-placed tail, will actually focus on agility as its metric for design. As such, the vertical tail will be placed to enhance mission performance rather than static stability. This tradeoff between performance and stability is certainly well known in the design community, so morphing provides an ability to alter that tradeoff during flight.

\subsection{Flexible wings}

A set of multi-jointed wings is critical to enabling mission capability for the Pterodrone. Certainly these flexible wings are essential to optimize flight performance; however, they are equally critical to achieve multiple modalities of locomotion such as walking and sailing. The existing platforms designed by the research team that use limited types of joints will be leveraged to rapidly design the novel vehicle for this project. In each case, a pair of joints is incorporated which are similar in nature to a shoulder and elbow.

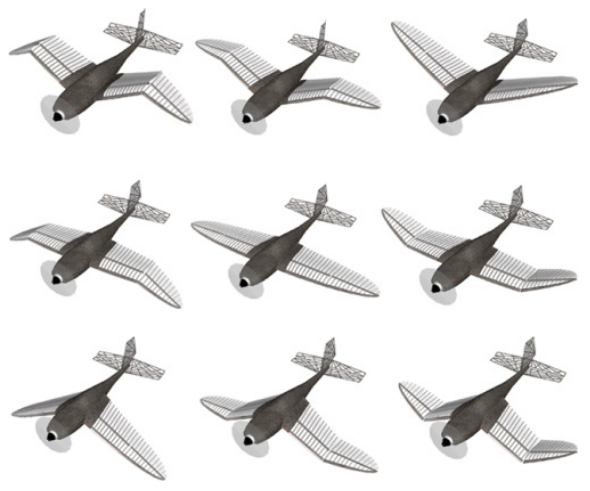

Figure 4: Variations in dihedral.

The vertical angle of the wings can be altered by rotating about a pair of joints at the inboard and mid-span locations. These joints allow independent change to the dihedral angle for both inboard and outboard sections on each wing. The pair of joints actually allows a wide range of configurations as shown in Figure 4.

The horizontal angle of the wings can also be altered using these joints. This angle, known as the sweep, is varied independently on the left and right wings for both inboard and outboard. Some representative configurations shown in Figure 4 demonstrate the range that can result from this multi-joint concept. 


\subsection{Materials and structures}

The payload capacity of the Pterodrone with a wingspan of $1.4 \mathrm{~m}$ and a mass of $0.4 \mathrm{~kg}$ allows us to proceed in the most direct manner possible. A combination of propulsions will be utilized for the vehicle to power the various modalties of locomotion. The power source for the propeller will vary based on size and weight. A gas engine will suffice for systems to supply sufficient thrust. The fuselage is modified as necessary to receive servomotors near the front, with axes of rotation collinear, horizontal, and perpendicular to the fuselage axis. The servos are modified to allow for continual rotation, and each drives one wheelleg appendage. An electric motor will be the primary source for the walking and sailing. Such motors, which are also very mature and easily available, have sufficient power to command the alterations required for the locomotion in these configurations. Additionally, the electric motors are quiet and thus appropriate for mission profiles that require walking or sailing to position a sensor close to a target.

A flexible wing is designed that will provide the necessary flight characteristics for the vehicle. As the wing parameters are entered, the graphical user interface displays in real time the top, side, and front projections of the wing. The program also outputs $2 \mathrm{D}$ aerodynamic estimates and key geometry features. This provides the user with theoretical feedback concerning the performance of the wing design.

A structure composed of several materials will be used for the construction of the Pterodrone. This approach will build upon the vast experience of the University of Florida team at building aerial robots to tailor the design to the needs of each individual element using a mixture of carbon fiber, Kevlar, and nylon. The carbon fiber will be prevalent throughout the structure. The process results from a composite cloth molded over tooling board. Detailed shapes, including complicated curvatures, are constructed by carving the molds using a CNC machine at the University of Florida. Finally, a piece-wise fabrication process will assemble the vehicle.

\subsection{Propulsion}

A combination of propulsions will be utilized for the vehicle to power the various modalities of locomotion. A single system would be ideal to reduce complexity; however, the on-board payload is sufficient to allow multiple systems of which each is optimized for the diverse maneuvers.

A gas engine will be the primary source of propulsion during flight. These engines are quite mature and indeed are routinely used and available through numerous vendors. They generate extremely high levels of thrust and will easily accommodate the size and weight of the vehicle.

An electric motor will be the primary source for the walking and sailing. Such motors, which are also very mature and easily available, have sufficient power to command the alterations required for the locomotion in these configurations. Additionally, the electric motors are quiet and thus appropriate for mission profiles that require walking or sailing to position a sensor close to a target. 
The issue of flapping will initially be avoided for this project. Obviously biological flyers, including pterosaurs and bats, use flapping as their primary means of flight; however, mechanisms for achieving sufficient magnitude and frequency of flapping for flight do not yet exist on this scale. A sacrifice of biological optimality is proposed to ensure an operable vehicle will be delivered.

\subsection{Avionics}

The fundamental mission for the vehicle is transporting sensors to a region of interest. As such, payload is an integral consideration in the design. A payload bay is included within the fuselage that will be able to house several tens of square centimeters of avionics. Additional instruments for surveillance can be mounted externally along the wings. Finally, the vertical tail presents a large area onto which sensors may be mounted.

Vision will be the initial type of sensing to be instrumented in the baseline vehicle. A forward-looking camera will be instrumented to provide real-time images. Additional cameras can easily be installed that look downwards or sideways to provide surveillance. These cameras can provide color images, which have already been shown as sufficient for information related to waypoint navigation, obstacle detection, state estimation, and scene reconstruction [8].

Also, sensors may be mounted to be actively controlled. The movements of the wings and vertical tail present obvious opportunities to direct the sensor towards a region without altering the flight path. Such sensing capability may be important for video or acoustic sensing along with radar. Additional sensors that relate state information about the vehicle will be included with the autopilot. These sensors include angles from magnetometers, angular rates from gyros, accelerations from accelerometers, position from GPS, and altitude from pressure sensors.

\section{Multimodal locomotion}

\subsection{Flight dynamics}

To examine the effects of the vertical tail placement in Pterodrone, Athena Vortex Lattice (an aerodynamics prediction code created by Mark Drela at MIT) was used to find the trim conditions of a MAV model [9]. For simplicity, the model used was a previously studied and successfully flown aircraft [10]. The only alterations were made on the vertical tail, making it a simple rectangle with the same surface area as the original, and adjusting its vertical and longitudinal placement. Furthermore, results were found at a wide range of velocities, so the results could be normalized to the velocity that it would require creating 2 Newtons of drag on the aircraft. In this manner the performance effects could be compared assuming a constant power plant that could provide only a maximum of 2 Newtons of thrust. Additional analysis showed similar results for a constant power model of propulsion that is common for propeller systems. 


\subsubsection{Agile turning}

The results show a marked improvement in the allowable turning radius when flight conditions are normalized to a constant drag. Results from this code also include the changes in the sideslip angle, angle of attack, and body axis stability derivatives. The vertical tail has a significant effect on turning; consequently, varying the location of that tail will vary the turn characteristics, which are obviously critical to agile maneuvering. In particular, a smaller radius turn, and correspondingly, a higher rate of turn can directly correlate to operating amongst denser obstacles.
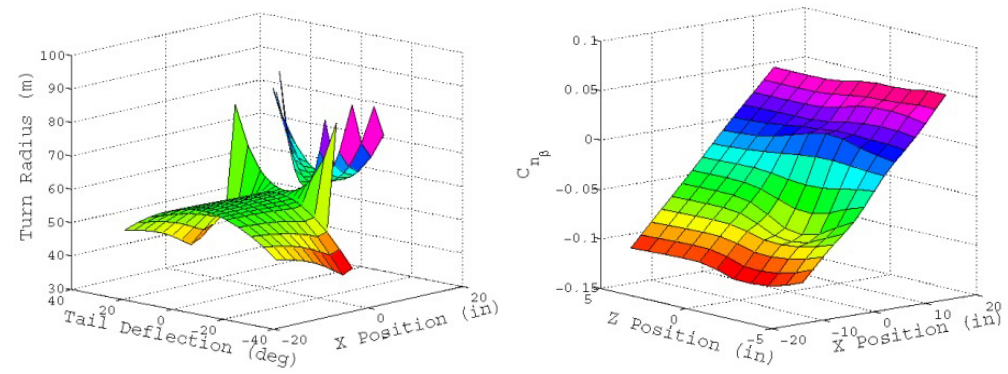

Figure 5: Reduction in turn radius as vertical tail is deflected when placed forward of center of mass, but accompanied by instability in yaw.

As expected, the derivatives show the appearance of lateral instability with a forward-placed vertical tail, but such instability can be controlled using modern control techniques while the vehicle could still benefit from the improved maneuverability resulting from unstable dynamics.

\subsubsection{Sensor pointing capabilities}

Additional aerodynamic analysis of wing configurations in conjunction with a forward placed vertical tail demonstrates a phenomenon by which the ailerons and morphing could be used to compensate for a vertical tail deflection.
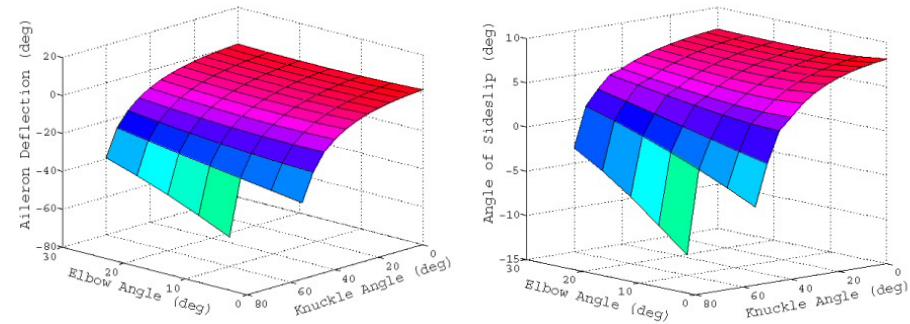

Figure 6: Trimming at various sideslip angles using wing sweep and aileron deflection with fixed vertical tail deflection. 
The combination of morphing and aileron deflection allows the vehicle to compensate for both the roll and yaw moments produced by an offset vertical tail deflection. If the vertical tail position is allowed to vary in both the longitudinal and vertical directions, then the surfaces could be used together in greater combinations to produce a wider variety of trimmed conditions. Increased freedom of trimmed flight conditions could thereby yield a vehicle with a greater ability to point a vehicle fixed sensor towards the region of interest.

\subsubsection{Vertical landing on the wall}

Walls are ubiquitous throughout urban environments, and so can present desirable vantage points for surveillance. The Pterodrone could slink up a building and perch there for hours or days, monitoring the structure or the terrain below. As such, the ability to perform sensor emplacement on a vertical surface is an important aspect to agility that directly relates mission capability. Such agility is well known among pterosaurs for climbing vertical cliffs with their highly recurved claws on hands and feet, so they present a clear source of biological inspiration. For suction on a vertical wall, a retracted tridactyl toe would be added to each rear wheel of the Pterodrone mimicking the gecko foot, christened here wheel-pod. It has a dual function, a wheel for takeoff and a foot for terrestrial locomotion, a contraption never used before. The transverse ridge and special adhesive on lower surface of the leathery toe of the wheel-pod would enable the Pterodrone to stick to the vertical wall and function as an additional brake during landing.

The dynamic motion related to vertical landing is a pitching maneuver. Essentially, the Pterodrone must rapidly pitch so the wings and fuselage are able to contact the vertical surface. The pterosaurs move their wings forward and down to initiate this rapid pitching [7]. The baseline model is thus altered to introduce variations in wing sweep and dihedral as design parameters correlating to the forward and down motion. After landing on the wall, a wing retraction mechanism improves the navigation of the vehicle through complex obstacles, as well as its terrestrial stealth and ability to enter small openings.

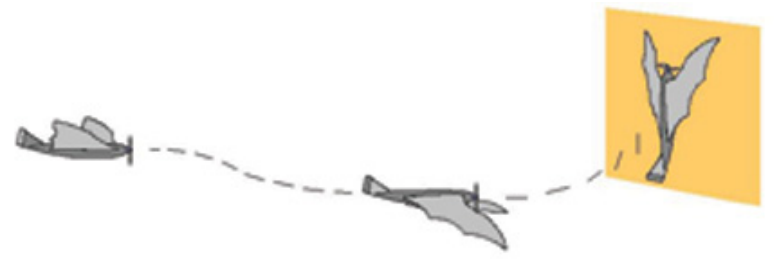

Figure 7: Representative maneuver for vertical landing on wall.

\subsection{Walking dynamics}

Several methods of terrestrial locomotion could be implemented in the design $[11,12]$. The first generation design favors simplicity of implementation over locomotive speed. Building a pure walking robot requires many joints, 
increasing complexity of design and movement and the weight of the vehicle, thereby making flight and sailing much more difficult. Thus, the first generation design attempts to retain as many of the advantages of the advantages of walking as possible while incorporating parts with multiple utilities to reduce the complexity. This design requires only two joints on each wing (shoulder and elbow) keeping complexity and weight to a minimum. The design also uses the rear wheels that are necessary for takeoff and the morphing head position used for improved flight control as integral parts of its walking motion, as well. The foot of the wheel-pod, on the other hand, would be useful during terrestrial locomotion by conferring grip and stability.

The first design uses wheels in the rear of the vehicle and morphs its wings to walk on its 'elbows.' During walking the shoulder joint permits the arms to move along the direction in which the vehicle is pointed [7]. Thus, the walking motion is more akin to a soldier crawling on his elbows and dragging his legs behind. In this sense, the design continues a biological-inspired approach since natural systems like the pterosaurs also use joints for walking. The concept designed for this vehicle adopts that that pterosaur-inspired approach as shown in Figure 8 .
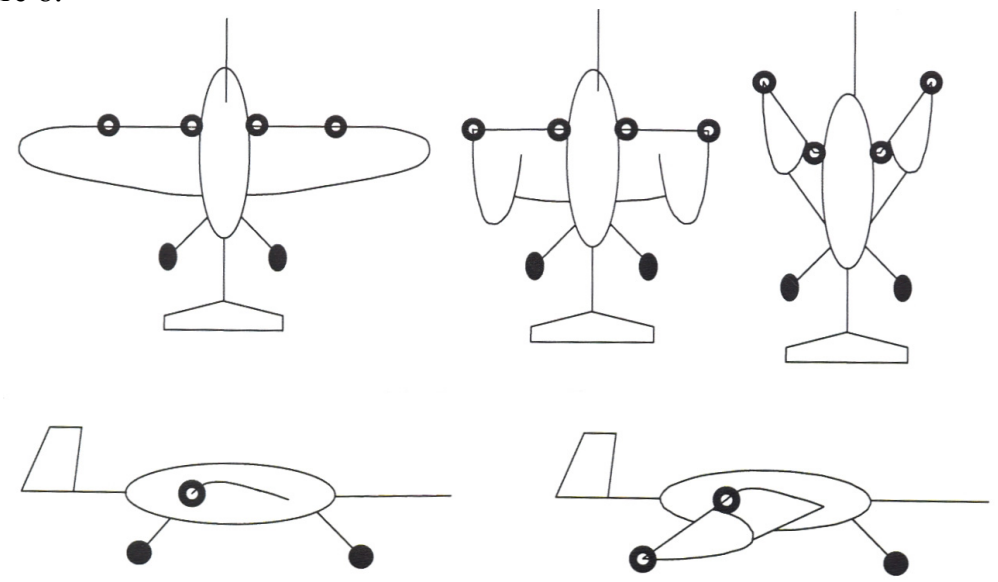

Figure 8: Top view (top) and side view (bottom) of flight to walking stance transformation.

\subsection{Sailing dynamics}

Pterosaurs could glide on the water surface in the downwind direction where the cranial sail in conjunction with two upright outer wings performed like a threemasted sailboat (Fig. 9).

The cranial sail might have served as a jib and a front rudder to steer the animal [5] as the ventral surface of the animal is rounded and streamlined without any keel or hull, the inner wings and hindlimbs served as multihulls and stabilizers like those catamarans [13]. The wing action of pterosaur is used to 

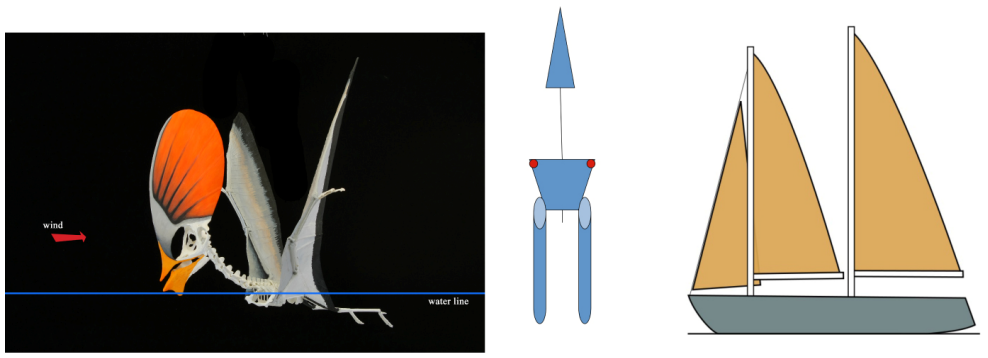

Figure 9: Left, restoration of a sailing Tapejara that mimics a two-masted schooner where the cranial sail acts like a jib. Tapejara could sail faster than the prevailing wind during foraging in upwind sailing by keeping the wings in upstroke position. Center, the hull of Tapejara had a catamaran design. Right, the sails of Tapejara resemble those of a two-masted schooner with a jib.

model the aquatic Pterodrone. Paleontologists have long noted that pterosaur wings were like sails, being membranes that could flex in either direction. The resemblance may prove to be more than passing: our research suggests the piscivorous reptiles sailed the seas as well as the skies during their extended reign for 160 million years [5]. We reconstructed the sail and cranial crest of Tapejara and made a series of models to examine its motion, then analyzed its aerodynamics and hydrodynamics with a biomechanical computer simulation. The sails of Tapejara resemble those of a two-masted schooner with a jib where the huge membranous head crest functions like a jib. The basic design of Tapejara is a cross between Wiebel (triple wind-surfing boards) and two masted schooner. Wiebel is the fastest sailboat in the world. Its hull has catamaran-like design with two lateral surfboards and a central aft surfboard. The hull of Tapejara resembles that of a Wiebel with a trimaran design. The sternum of Tapejara resembles the central surfboard, which is positioned forward, whereas two legs represent lateral surfboards. Thus the reptile's sternum and legs would have contacted the water much as a trimaran's hulls do. Tapejara could glide across the surface with minimal effort, keeping their wings upright to catch a breeze, probably for short distances between bouts of fishing.

For all types of hulled craft, the first design concern is the roll stability of the craft while submerged. The most important stability measure is a comparison between the heeling and righting moments. However, the current design has no aerodynamic surface above the water; consequently, very little heeling moment would be created, even in a stiff wind. Thus, the righting arm curve can be analyzed as a good indicator of the crafts stability as shown in Figure 11. The stability can be augmented using a pair of methods. The first is to lower the center of gravity so the heavy components such as the batter are lower to create a larger righting moment. The second method increases the hull size to increase the residual buoyancy moment. The morphing wings could also balance the vehicle, in a way that ships can not, by morphing the wings to move the center of gravity, thus increasing the righting moment. 

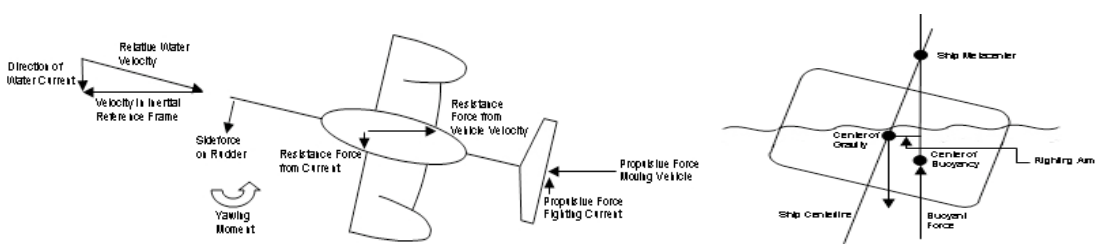

Figure 10: Left, balance of forces and moments; right, stability analysis.

Once the design is proven stable, then the performance will be analyzed, with the maximum relative speed, acceleration, and turning rates being the most important. The associated forces and moments used to determine performance are shown in Figure 10. Note the propeller remains out of the water to avoid inefficiencies with submerged propulsion.

\section{References}

[1] Abdulrahim, M., Garcia, H. and Lind, R. 2005. Flight characteristics of shaping membrane wing of a Micro Air vehicle. Journal of Aircraft, 42, pp. 131-137.

[2] COM-BAT Research Project: University of Michigan. Online. http://www.ur.umich.edu/0708/Mar17_08/02.php

[3] Lambrecht, B. G. A., Horchler, A. D., and Quinn, R. D. 2005. A small insect inspired robot that runs and jumps. Proceedings of the International Conference on Robotics and Automation, ICRA, Barcelona, Spain, pp. 3348.

[4] Chatterjee, S. and Templin, R. J. (in press). The flight dynamics of Tapejara, a pterosaur from the Early Cretaceous of Brazil with a large cranial crest. Proceedings of the National Academy of Sciences of the United States.

[5] Chatterjee, S., Alexander, D. E., Lind, R., and Gedeon, A. 2009. The sailing performance of the crested pterodactyloid Tapejara from the Early Cretaceous of Brazil. GSA Abstracts with Programs, 41(7), 685.

[6] Chatterjee, S., Lind, R., Gedeon, A., and Roberts, B. 2008. Pterodactylinspired unmanned aerial vehicle with multimodal locomotion. GSA Abstracts with Programs, 40(6), pp. 394.

[7] Chatterjee, S. and Templin, R. J. 2004. Posture, locomotion, and paleoecology of pterosaurs. Geological Society of America Special Paper, 376, pp. 1-63.

[8] Kehoe, J. J., Causey, R. S., Abdulrahim, M., and Lind, R. 2006. Waypoint navigation for a Micro Air Vehicle using vision-based attitude estimation. The Aeronautical Journal, 110, pp. 821-829.

[9] Drela, M. and Youngren, H. 2000. AVL-aerodynamic analysis, trim calculation, dynamic stability analysis, aircraft configuration development. Athena Vortex Lattice, v. 3.26. Online. http://raphael.mit.edu.avl/ 
316 Design and Nature V

[10] Grant, D. T., Abdulrahim, M. and Lind, R. 2006. Flight dynamics of a morphing aircraft utilizing independent multiple-joint wing sweep. American Institute of Aeronautics and Astronautics, pp. 6505.

[11] Alexander, R.M. 1992. Exploring Biomechanics: Animals In Motion. W. H. Freeman, New York.

[12] Todd, D. J. 1985. Walking Robots: An Introduction to Legged Robots. Kogan Page Ltd., London.

[13] Marchaj, C. A. 1979. Aero-Hydrodynamics of Sailing. Dodd, Mead \& Co., New York. 